\title{
Mineralogische Chemie.
}

\author{
I.
}

Beiträge zur nühern Kenntriss backender Sleinkohlen, vorziiglich deren Anwendung zum Zusammensintern slaubiger Erse und Hillenproducte betreffend,

$$
\text { von }
$$

W. A. LAMPADIUS.

varwort.

Backende Steinkohlen nennen ivir bekannthch diejenigen Schwarzkohlen, welche sich schon bei mïssiger Erhityung erweichen; selbst bei dem Verbrennen auf Rosten halh zusainmen schmelzen, und bei der Verkohlung in ganz zusammen geflossene, jedoch aufgeschwollene und poröse Coaks übergehen. Zwei Ursachen veranlassten mich, zwei dergleichen Sorten sïchsischer Steinkohlen in Arbeit zu nehmen, nimblich 1) wollte ich die Natur dieser Steinkohlen, vorzüglich in Ilinsicht ihrer bakkẹden Eigenschaft näher erforschen, und z) lag mir daran, diese ihre Eigenschaft zun Zusammensintern fein zertheilter Erce and Fluggestübe, die in Schachtölen verschmolxen werden sollen, nut\%bar in das Leben treten zu lassen, welcher Anwendung ich bereits in Erdmann's Journal für techn. und ökonom. Chemie B. II. S. J15 kurz erwähnt habe.

1) Versuche über die Natur backender Steinkohten.

Ich verschaffte mil $z$ diesen und allen nächstfolgenden chemischen Bearbeitungen dieser. Schwarzkoblen zwei Arten derselben, welche ich mit No. 1 und 2 bezeichnen will.

No. 1. Pechkohle von Oberholndorf bei Zwickau. Sie wurde mir durch den dortigen Factor des Steinkoblenwerkes, Journ. f. prakts. chemie. VII. 1. 
Herrn. Hof fman n, mit der Bezeichnung zugesendet: „Steinkohlen von dem jetzt am meisten in Schlage stchenden, sogenannten Pechkohlenlütz im jungen Wolfganger Felde." Es war grösstentheils Pechkohle, mit wenig cingemengter Schieferkohle, und kaum bier und da merklichen Anflügen von Faserkohle, mithin eine Kohle von der besten Beschaffenheit dortiger Flötze.

No. 2. Gemenge von Pech- und Schicferkohle von WühIcn unweit Dresden. Der Uebersender, Herr Factor Lindig. zeigte mir an: „Diese Kohle sei aus den südlichen Banen der königl Döhlener Steinkohlenwerke, in der Nähe des Dühlener Kunstschachtes gewonnen; sie backe selir gut, und habe den geringsten Erdengehalt unter den dortigen Steinkoblen."

A. Coaksbereitungsversuche.

Es wurden 20 Unzen 6 Drachmen 40 Gr. $=10,000$ Gran, von jeder Kahle gräblich zerstossen, in stehenden, mit Deckeln versehenen cylindrischen Büchsen dcm Verkoblungsprocesse uncrworfen.

No. 1 gab 6160 Gran $=61,6$ Gewichtsprocent von ziemlich lockern, etwas porösen Coaks, in ein cylinderförmiges Stück ausammen gesintert. Der Coakscylinder nahm $1 / 8$ mehr Raum, als die zu dessen Bildung verwendeten rohen Steinkohlen eiu. Der zerstückte Cylinder zeigte sich durchgiungig gut verkohlt, und die Coaks waren von vorzüglicher Beschaffenheit in Hinsicht auf Glanz, Farbe u. s. w.

No. 2 gab 6840 Gr. $=68,4$ Gewichtsprocent Cooks, ebenfalls zu einem Stück zusammen gebacken. Der Cylinder nahm $1 / 10$ mehr haum ein als die verweudeten rohen Steinkohlen. Die Coalis waren von guter Beschaffenheit, nur etwas dichter wie die von No. 1 und hier und da nur gesintert.

\section{B. Einüscherungsversuche.}

Die gröblich zerstückten Coaks beider Steinkohlensorten wurden anf facben Thonscherben vertheilt, unter einer grossen, stark rothglüheil len Muffel mit Vorsicht völlig eingen̈schert. Sic mussten, um dieses zu erreichen, ein Mal durchgeglühet, and schon halb eingeüschert, aufgestossen werden. Unterlïsst man direses, so bieibt Ieicht ein Kern von unverbrannten Coaks zurück. 
No. 1. 6160 Gran Coaks gaben 416 Gr. einer feinen gelblich grauen Asche; das ist anf die rohen Steinkohlen berechnet 4,16 p. C. Asche und $5 \mathbf{5 7 , 4 1}$ p. C. Kohlenstorf.

No, 2. 6804 Gran Coaks gaben 1200 Gran einer grauen, hier und da etwas gesinterten Asche; d. i. auf die roben Steinkohlen berechnet 12 p. C. und 56,04 Kohlenstoff.

Die Bestandtheile der Aschen dieser Kohlen siod hinlänglich bekannt, nämlich Thonsilicat, kohlen-, schwefel- und phosphorsaurer Kalk, Eisen- and Manganuxyd, nebst Sparen von Talkerde. Die Steinkohlen der Zwicknuer Formation enthalten etwas mehr kohlensauren, die der Dühlener etwas mehr schwefelsauren Kaik.

C. Flïchtige Producte der Verkohlung.

Es wniden von jeder Sorte der Steinkoblen No. 1 und 2 10,000 Gran gröblich zerstossen, in liegenden gusseisernen $\mathrm{Cy}-$ linderretorten mit vorgelegtem Kühlapparat, welcher mit einem Gasometer in Verbindung gesetzt war, verkohlt, und man erhieit:

a) Aus der Koble No. 1.

Gas ïberraupt . . . . . . 7950 Par. c. z.

Nach dem Auswaschen mit Kalliwisser ver-

blieben Leuchtgas . . . . . $\quad$. $7399 \quad$ -

Ist zu berechnen absorbirtes kohlens. Gas 561 -

Das Leuchtmas war vortrellich und gab ein rauch - und geruchfreies Weisslicht.

Das Destillat des Kühlapparates wog 1997 Gr., und wurde durch die Filtration zerlegt in:

6\$0 Gran Theer $=6,8$ p. C. und

1317 - Theerwasser $=13,17$ -

b) Die Steinkohle No. 2 gab:

Gas überhanpt . . . . 7340 C. z.

Darin Leuchtgas . . . . 6790 -

Kohlensaures Gas . . . $.550 \quad-$

Das Leuchtgass war ebenfalls von gehöriger Güte.

An Destillaten wurde erlalten:

614 Gran Theer $=6,14 \mathrm{p} . \mathrm{r}$.

$1325-1$ - 'lieerwasser $=13,25$ -

1939. Gran Destillat. 
4 Lampadius, über backende Steinkohlen.

D. Harz - oder Bitumengehalt der Steinkohlen.

Is ist von verschiedenea Naturforschern angenommen worden, dass sich in den Steinkohlen keine barzige Substanz oder keiu Bitumen rorfinde. So heisst es in Karsten's lehrreicher Eisenhüttenkunde, Thl. II. S. 282, Uass sich Bitumen weder in der Braunkohle noch in der Steinkohle finde, sondern nar ein Product der Destillation sci. \$. 315 desselben Theiles des Werkes heisst es: „Das überwiegende Verhältuiss des Wasserstoffs zum Sauerstoff in den Backkohlen bewirkt, dass die Steinkohle in dem Angenblicke, wo die Zersetzung eriolgt, in einen halbgeschmolzenen Zustand übergeht, so dass die erweichto und zum Theil teigartig geworlene Masse durch die sich entwickelnden Dämpfe und Gasarten in die Höbe getriehen und nach allen Richtungeu ausgedehnt, und oft blasenförmig aulgeblähet wird." Wenn es nun auch keinem $Z$ weirel unterworfen ist, dass das im Destillate der Stein - und Braunkohlen befindliche, in flüchtigem Oele aufgelüste Schwarzharz eben so grut ein Product als im Destillate der Höler ist, so haben mir doch öfter wiederhoite Prüfungen gelehrt, dass in mehreren Steinkohlenarten, namentlich in den backenden und sinternden Schwarzkohlen, ziemlich viel Bitumen, ich will es natürliches Steinkohlenhary nennen, enthalten ist, und dass sich auch Spuren von demselben in andern fossilen Kohlenarten vorfinden. Ich babe bereits in meiner Abhandlung über Anthracit, s. dieses Journ. B. IV. S. 414, angeführt, dass ich mich des liquiden Kohlenschwefels (Schwefelalkohols) zur Prüfung der Steinkolulen auf einen Fangehalt bediene, und es wird bier am rechten Orte sein, das bei dieser Prülung von mir gebrïuchliche einfache Verfahren genauer anzugeben. Lm einige Versuche über die Eigenschaften des Steinkoblenharzes anstellen zu können, wählto ich zur Extraction desselben, aus den Oberhohndorfer und Dühlener Pechkohlen, No. 1 und 2 eine grössere Quanität, wie gewöhnlich zu dergleschen Aualysen angeweudet wird. Eg wurden nämlich 1000 Gran von jeder Steinkoblensorte in das feinste Pulver zerrieben, und in einem Glaskolben mit 6000 Gran liquidem Kobilenschwefel ${ }^{*}$ ) übergossen und gut gemengt.

*) Ich hatte zum Beluf dieser Untersuchung einige Pfd. SchwePelalkohol von meinem geelirten Freunde, Herrn Prof. Beichard in Döblen, erhalten. Er war so vollkommen rein, dass er bel der 
Hierauf wurden die Kolben mit Blase verschlossen, und in der Temperatur eines missig geheizten Zimmers 3 Tage lang hingestellt, während welcher Zeit der Inhalt derselben zuweilen aufgeschüttelt wurde. Schon am ersten Tage Abends sahe man den Schwefelaikohol sich dunkel weingelb fïrben, und ein wenig opalisiren. Letzteres rührte, wie ich spater faml, von einem geringen, mit der Lusung gemengten Wassergehalt der Koble her. Nach Verlauf von 3 Tagen filtrirte ich die dunkel weingelbe Lüsung in ein Siöpselglas ab, und süsste day rückständige Steiukohlenpulver noch mit etwas Schwefelalkohol aus. Ich brachte dasselbe yon Neuem in den Kolben, und wiederholte dieselle Extraction mit noch 3000 Gran selwwefelalkohol. Die erhalterıe Lösung wurde in dem von mir B. IV. S. 388 dieses Journals beschriebenen Apparate auf einer tarirten Glasschale allmäblig eingedampft, wobei ich 8000 Gran des Schwefelalkohols reichlich wieder erhielt.

Aus der Lösung der Steinkohle No 1 erhielt ich 47,7, und nus jener der Steinkoble No. $2,40,1$ Gran natürliches Steinkohlenhar:; das ist mithin aus No. 1 gegen 5 , und aus No. 2, 4 p. C. Es sei hier noch bemerkt, dass sich, wic ich später bei kleinen Versuchen gefunden habe, der Schwefeläther ebenfalls zu dieser Extraction gebrauchen lïsst, und dass bei einer nochmaligen Behandlung des Rückstandes, van der zweiten Extraction noch ein keiner Autheil von Harz ausgerogen wird.

Eigeuschaften des natürlichen Steinkohlenharzes.

Es ist von schu:arobrauner Farbe, undurchsichtig, nur in dünnen Lamellen durchschteinend; nicht gan\% so dunkel gefürbt, wie künstlich producirtes Steinkohlenharr; es ist sowohl kalt als erwärmt geruchlos; erscheint geschmoken glasglänzend, und von muschligem Bruch.

Das spec. Gew. ist ungeführ dem des Wassers gleich. In der Wärme des Sandbades von $70^{\circ} \mathbf{R}$. gerïth dasselbe ool kommen in Fluss.

Auf einem messingenen Lüfel über der Spiritusflamme er-

Verdunstung keine Spur einer fremdartigen Suhstanz zurückliesa. Die oben beschriebenen Versuche liess ich, um nicht so viel Schwefelalkohol auf einmal zu verbrauchen, auf einauder folgen. 
hitzi, entzunndet es sich und brennt mit russiger Flamme; hinterlässt dabei keinen Rückstand.

Im Schwefelalkohol löst es sich leicht schon in der Kälte auP. Die Lösung ist bei geringem Harzgehalt lichtbraun und durchsichtig, wird aber bei stärkerem Gehalt schwarzbraun, und zuletzt bei völliger Sättigung dickflüssig syrupartiğ. In diesem Zustande hatten 10 Gran Schwefelalkohol 19 Gran von dem Steinkohlenharze aufoenommen. Ein mässig erwärmtes Messingschälchen worde mit dieser Lösung sogleich bei schneller Verdunstung des Lösungsnittels lackirt.

Der Schucefeläther, der absolule Alkohol, das Terpenthinand Leinöl lösen das Harz, letztere beide znerst durch Beihülfe der Wärme auf.

Durch die Kochung mit Wasser wurde nichts ans dem Harze ausgezogen.

- Fs besitzt allem diesem za Folge die Substanz alle Eigenschaften eines Hartharzes, and verdient daher mit Recht den Namen natürliches Steinkohlenharz. Ob dieses Harz in Varietïten in verschiedenen Steinkohlenarten vorkommt, müssen fernere Bearbeitungen der Steinkohlen lehren. Die aus den hier in Rede stehenden Steinkohlen No. 1 und $z$ erhaltenen beiden Antheile dieses Harzes, verhielten sich gleich in ihrem nussern Ansehen und ihren chemischen Eigenscharten. Hüchst wahrscheinlich verdankt dieses Harz seinen Urspruag den bei der Steinkohlenbildung mit versenkten harzigen Holzarten, und ist nur ein durch den Steiakohlenbildungsprocess ungeëndertes Baumharz.

E. Coaksbereitung aus entluarzten Steinkohlen.

Es wurden 10 Quent. $=600$ Gran der beiden Steinkoh. lenpulver, welche auf den Filtern nach der Extraction ibres Harzgehaltes zurückgebliebrn waren, in zwei Probirtuten mit Deckeln belegf, zur Coaksbereitung zwischen glühende Kohler eingesetzt. Als Gegenprobe übergab ich demselben Feuer ir 2 Probirtuten 600 Gran von den Steinkohlen Vo. 1 und 2 , im rohen Zustande, aber ebenfalls gan $\approx$ fein pulrerisirt.

Bei dem Hergange der Verkohlung wihrend einer Feucrung von $11 / 2$ Stuaden, war nichts besonders $A$ bweichendes be dem Verbalten der vicł Verkollungsgefüsse wahrzunehmen. 
Sie liessen unter den Deckeln zuerst Dämpfe darch, welche sich später entzündeten, und sobald dieses Flammen aufbörte, wurde der Process unterbrochen.

Nach der Erka!tung und dem Zerschlagen der Verkohlungsgefïsse zeigte sich ihr Kohleniuhalt von sehr rerschiedener Beschaffenheit.

Die Coaks von dem rohen Steinkohlenpulver waren wie oben bescbrieben, vōllig glïnzend, etwas blasigt, aufgeschwollen und in einem stück rusammenhïngend.

Die Coaks von dem entharzten Steinkohlenpulver hingegen, waren ganz schwach gesintert, matt von Ansehen, zwischen den Fingern leicht zerreibbar, stark abfärbend; weder blasig noch aufgeschwollen *).

Es ist daher wohl keinem Zweifel unterworfen dass diesem Harzgehalt die erste Erweichung der Pechkohlen bei der Coaksbereitung zuzuschreiben ist. Bei der fortschreitenden Verkohlung wird dieses Har\% wieder zersetzt und hinterlisst eine leichte starkglänzende Kohle, welche sich mit der übrigen Coaksmasse innig vermengt. Uebrigens geht bei dem Verkoh lungsprocesse ausserdem forthauernd die Bildung von Schwarzharz und flüchtigem Steinkohlenöl (Theer) vor sich.

F. Versuche über die Zusammensinterung staubiger Eræe und Hüttenproducte durch die Steinkollen Nr. 1 und 2.

Es beschäftigten mich dergleichen Versuche im Kleinen bereits seit dera Jahre 1826, und ich habe derselben in Erdmann's Journ. f. techn. und $0 \mathrm{k}$. Chem. B. II. S. $40 z$ u. 515, so wie in meinen Vorträgen über Hüttenkamle mehrmals $\mathrm{Fr}$ wähnung gethan. Durch die in dem Repertory of patent inventions 1827 bei uns in Jahre 1828 bekannt gewordcnen Mittheilungen, dass Jefferies gröblich gepochte Erze und metallhaltige Producte mit Steinkohlenhlein sintere und die gesinterten Massen verschmelze, wurde ich om so mehr veranlasst, diese Versuche, namentlich für das sächsische Hïttenwesen berechnet, fortzusetzen. Nachdein ich nun durcb mehrere wie-

*) Herr B. C. R. L a mpadius hat die Gïte gehabr, mit Proben von jeder Coaksorte sowohl als auch von den steinkohten Nr. 1 u. 2 Itud vou dem extrahirten Steinkohlenharze zu ïbersenden, wodurch ich Gelegenheit erhielt, mich von deren Beschaffenheit, die vollkommeu den obigen Augaben entspricht, zu überseugen. Erdmanı. 
derholte Versuche mit verschiedenen Arten sïchsischer Schwarzkohlen zu der Ueberzeugung gekommen war, dass nur die begten Backkohlen sich zL der in Rede stehenden Bindung der Erze eignen, so erbat ich mir, wie oben gesagt, dergleichen Steinkohlen von den Vorstehern der Steinkohlenwerke und unternahm mit diesen, bereits oben unter Nr. 1 und 2 bezeichneten, in dem letztverflossenen Halbjahr mehrere genauere Versuche mit kleinern und grössern Partien der Gemenge. Um Weitläufigkeit zu vermeiden, werde ich hier nur das Verfahren bei diesen Versuchen angeben, und, mit Weglassung aller Wiederholungen, die durch die Versuche erlangten Hauptresultate mittheilen.

Ich batte bei der Anstellung derselben vorzüglich mein Augenmerk auf die Zinner $\approx e$, welche als feine Schliche über Schachtöfen nur mühsam zu verschmelzen sind, gerichtet; jedoch nahm ich auch auf dürre staubartige Silberer\%e, so wie auf Flugstaab mit Rücksicht. Den Zinnstein zu den neuern Versucken erhielt ich vermöge meines Gesuchs durch hohe Verordnung des königlichen Oberbergamtes zu Freiberg von Altenberg, und das Dürrerz, so wie den Flugstaub durch das königliche Oberhültenamt von der Halsbrückner Schmelzhütte.

Der Zinnstein war gehörig durch Röst- und Wascharbeiten \%u ganz feinem lichtbraunem Schlich aufhereitet und ein Maass 䉼, welches 100 Gewichtstheile Wasser fasste, nahm 290 Theile Zinnstein auf.

Das Dinrers war ein silberarmes, gepocutes, quarzigschwerspïhiges von der Grube Churprinz Friedrich August. Das obigc Maass wog 210.

Der Fluystaub von granschwarzer Farbe war ans der Gestübekammer eines Bleiofens, und theils staubig, theils etwas gesintert. Zerrieben und gröblich durchgesiebt, wog ein Maass 110. Das gröbliche Pulver der Steinkohlen Xr. 1 wog im Verglcich mit 100 Wasser 81 und das der Steinkohlen Nr. 2,83 .

Die Sinterungstersuche im Kleinen worden in Probirtuten mit 10 Quenichen der Erze und des Flugstaubes angestelit. Ich vermengte dicse Substanzen mit Steinkohlenklein in abweichen-

*) Ich bemerke dieses Gewicht eines Maasses fiir diejenigen, welche im Grössern die zu vermengenden Substanzen statt zu verwiegen, vermessen wollen. 
den gewıgenen Verhältnissen, und liess sie in den bedeckten Probirtuten bei müssigem Feuer verkohlen und sintern. Mach beendigtem Versuch wurden die Probirtuten zerschlagen und ihr Inhalt wurde gewogen und nach dem äussern Verbalten beurtheilt.

Die grössern Versuche mit 2 bis 3 Pfand der Erze and des Flugstaubes wurden in einer cylindrischen Büchse von Schniedceisen $16 \mathrm{Z}$ oll hoch und $7 \mathrm{Z}$ oll weit, betrieben. Der Deckel dieses Verkohlungsgefässes ist tubulirt. Durch den Tubus entweichen die flüchtiggen Stofie bei der Verkohlung; anfänglich rauchend, und spitter flanmend.

Die kleinern Versuche liess ich vorangehen, um vermöge deren Ausfall, wenn sich derselbe günstig zeiğte, einen grüssern Versuch, durch welchen man grüssere Massen zur Beurtheilung erhielt, folgen zu lassen.

Ich fing mit dem Zuschlage von 10 Gewichtstheilen Steinkohlenklein auf 100 Theile der zu sinternden Substan\% an, und stieg von $10 \mathrm{zu} 10$ bis $\% \mathrm{ugleichen}$ Theilen der Steinkohle und des Erzes oder Flugstaubes, welches letztere Verbältniss nngefähr die Quantitat Coaks, die zu der Verschmelzung der in die gebildeten Coaks eingesinterten Sabstanz nüthig sein würde, ist. So würden \%. B. 100 Centn. Zinnstein mit 100 Centn. Steiukohle Nr. 1 verkohlt 161 Centu. zinusteinhaltige Coaks mit 57 Centn. Koblenstofgehalt geben.

Resuitate der Versuche im Kleinen.

a) Bei 10 p. C. Steinkohlenzuschlag *a zeigte sich bei keinem der Erze, eben so wenig bei dem Flugstaub Sinterung.

b) Bei 20 p. C. Zuschlag war bei dem Zinnstein eine schwache Sinterung wahrnehmbar; das Dürrerz und der Flugstaub bliehen noch pulvericht.

c) Bei 30 p. C. Steinkoh'enzuschlag war der Zinnstein bis zum Klümprigen gesintert; das Dürrerz zeigte einige, der Flugstaub norh keine 'Sinterung.

d) Bei 40 p. C. zeigte sich noch ziemlich dasselbe Verhältniss; jedoch fing auch der Flugstamb zu sintern an.

e) Bei 50 p. C. Steinkohlen erschien der Zinustein schon

*) Die steinkohlen N*, 1 u. 2 verhielten sich bei diesen Sinterungsversuchen gloich. 
theils in festen Stücken, theils in gesinterten Kernen; nicht ganż so gesintert das Dürrerz and der Flugstaub in erbsen - und linsengrossen Körnen, zurn Theil noch als lockeres Pulver.

f) Bei 60 p. C. Steinkohlen war die Partie des Zinnstiicks, welche die äussere Peripherie der verkohlten Masse bildete, schon fest zusammenhüngend, und die des Kernes noch in kleinern stücken; das Dürrerz zeigte sich theilweise in Brocken, theilweise als grobes Pulver. Das Fluggestübe verhielt sich dem Dürrerz ähnlich, jedoch waren die Stückchen leichter zerreibbar.

g) Bei 70 p. C. Steinkohlenzuschlag waren alle Substanzen völlig gesintert; nur mit dem Unterschiede, dass der Zinngtein fest; das Dürrerz mäsijg und der Flngstaub geringe zusammenhing.

b) bis k) Bei 80 bis 100 p. C. Steinkohlenzaschlag kamen zwar sämmtliche Besehickungen in einem zusammenhïngenden Stück aus den Probirtuten; ailein nur der Zinnstein war völlig hart und fest, härter noch wie gewöbnliche Coaks; dem ziemlich nahe kommend verhielt sich das Dürrerz; die gesinterte Masse des Flugstaubes hingegen liess sich noch mit den Fingern zerdrücken.

Resultate der Versuche im Grössern.

a) 3 Pfund Zinnstein und 3 Pfund Steinkohlenklein Nr. 1 gaben 4 Pfund 21 Loth festgesinterte Masse, grau, ziemlich dicht ohne Blasenrïume, ziemlich schwer zerschlagbar. Der Kern war etwas weicher. Bei der Verkohlung batte sich am Deckel des Verkohlungsgefiasses etwas arsenigte Sïure angelegt; anch roch die Flamme ein wenig arsenikalisch, woraus sich ergiebt, dass der Zinnstein durch diese Behandlung noch mekr gereinigl uerden kanr.

b) 3 Pfund Zinnsteia und 3 Pfund Steinkohlenklein Nr. 2 gaben 4 Pfund 30 Loth ebenfalls festgesinterte Masse. Alles übrige war wie bei Versuch a.

c) 3 Pfund Zinnstein und 1 Pfund Steinkohlenklein waren noch leidlich gresintert, doch weniger fest als a.

d) 3 Pfund Dürrery und 3 Pfund Steinkohlenklein Nr. 2 waren recht gut gesintert, jedoch weniger fest als bei Versuch a.

c) 1 Pfund Flugstaub und 1 Pfund SteinkohIcnklein Nr. 2 
gab nur theilweise eine ziemlich fcste Masse von noch ganz mattem Ansehen und leicht zerreiblich. Bei der Verkohlungwurde ein schwacher, arsenikalischer und schwellichter Geruch wahrgenommen; jedoch gab es keinen Anflug.

Es ergiebt sich nun ats vorstehenden Daten: dass staubige Erze und Hüttenproducte, um völlig zum Sintern gebracht zu werlen, ungefähr eben so viel Steinkohle als im Zustande der Coaks zu ihrer Verschmelzung nüthig scin würden, bedürfen, und dass diese Sinterung um so besser erfolgt, je specifisch schwerer die zu sinternden Pulver sind, oder was dasselbe ist, je weniger Rum sie, mit der Steinkohle gemengt, einvehmen. Miissige Zusammensinterungen, welche den Erren und dem Flugggestübe nur das ganz staubartige benehmen, erfordern 20 bis 40 p. C. gubacliender Steinkohlenarten.

(Als Zugabe zu diesen Versuchen führe ich noch an, dass, um 100 Gewichtstheile Zinnstein durch Steinkohlentheer zum Sintern zu bringen, 10 Theile des letztern nüthig sind. $100 \mathrm{Th}$. Dürrerz erfordern 12 , und 100 Theile Flugstaub 15 p. C. dieses Theers. Man erwürmt die pulverigten Substanzen in einer eisernen Pfame oder auf einer Eisenplatte, und rührt das Theer darunter, erhïlt die Masse einige stnnden über gelindem Feuer, und findet sie nach dem Erkalten in festen Stücken. Der Zinnstein ist auf diese Weise auch in ein vortreffliches Reductionsmittel eingehüllt).

G. Ceber die Ausfilhrang der vorstehenden Sinterangsmethoden in Grossen.

Wenn nun, wie ich hofen darf, die Resnltate vorstehender Versuche Veranlassing zu weitern Versuchen in Grössern aul den Hültenwerken selbst geben sollten, so würde meiner unmaassgeblichen Ansicht nach, dabei folgendermansen zu verfahren sein:

1) Komnt es darauf an, die staubigen Substanzen einigermaasen gegen das Verblasen $z u$ schützen, so wird es hinreichen, sie mit 20 bis 40 p. C. Lüsche ron Bachloblen zu mengen und sic mit andern Beschickungstheiten ant die Gicht der Schachtïfen zu geben. bas Gemenge wird ham wahrend des Niedergehens, und ehe es in der Forwgegend andangt, cinigermasen sintern. 


\section{Lampadius, über backende Steinkohlen.}

2) Eine schon bessere Sinterung wirl man erlangen, wenn man Erze oder Fluggestübe mit 40 bis 70 p. C. Steinkohlenklein in glühend gemnclıten Backöfen oder anch in Rüstöfen, zum Schluss der Küstung noch ein Mal stärker erbitzt, zum Sintern bringt.

3) Die beste Sinterung wird mit gleichen Gewichtstheilen Steinkohlenklein und staubiger Substanz, mit Berücksichtignng der Bcnutzung-der flüchligen Flammenstoffe der Steinkohlen vielleicht folgendermaassen vorzunehmen sein:

Man verbinde einen Verkohlungsgefässofen, z. B. einen Gasretortenofen oder einen aus eisernen Platten zusammengesetzten Kastenofen mit einen Flammenröstofen, z. B. mit deu Zinnerzröstofen, sintere in dem ersten das Erz mit dem Steinkohlenklein, und entzünde dabei die sich entwickelnden Gasand Theerdämpfe in dem anstossenden Rüstofen. Eine solche Vorrichtung ist vermöge des verschiedenen Locals mancher Modiflcationen fäbig. Es sei $\%$. B. ein Gasretortenofen mit $\mathbf{3}$ Retorten hinten an der schmalen Seite eines Flammröstofens angelegt, so lasse man aus den versehlossenen Enden der Retorten Rohre zur Ableitung der flüchtig̀en Biennstoffe in ein horizontalliegendes Hauptrohr münden. Dieses kann mit einem Hahne versehen werden, um nöthigen Falls angesammelte Flüssigkeiten abrulassen. Dieses Hauptrohr muss der Länge nach in der Hauer des Röstofens dicht an Feuerheerde eingelegt werden. Aus diesem Hautrohre mögen nun 6 bis 8 , ungefailir $1 / 4$ Zoll weite eiserne hühren in den Feuerheerd mïnden. Durch diese treten Gas und Theer aus uni kümnen, so wie die Verkohlung vorschreitet, leicht entzündet werden. Da man bereits mit Steinkohlengas allein Werkblei abgetrieken hat (s. Eramann's Journ. f. ok.u. t. Chemie, B. V. S. 206), so wird man um so mehr mit dem, mit den Theeräampfen noch vermengten Gase rüsten können. Die Stürke und Länge der Flamme wird, da es hier wie bei der Gasbeleuchtung auf rauchfreies Verbrenuen ankommt, weit hedeutender als bei dem blossen Gase erscheinen. Man wird sich bei einem solchen Rüsten alsdann nach der Zeit, in welcher die Steinkohlenflamme erscheint, richten müssen, kann übrigens in demselben Röstofen vor oder nach und auch wührend der Erscheinung der ent- 
La mpadius, Unters. eines Mineralwassers v. Elba. 13

zindlichen Verkohlungsproducte, mit den gewöhnlich gangbaren Brenumateriai rösten.

So, meine Ich, kann man in Ifem Retortenofen sintern, in dem Müstofen daneben rösten, und die gesinterten Massen in Schachtölen verschmelzen.

Wenn nun ein gegebenes Qunntum von Steinkohlen in Retorten soll vercoakt werden, so gehïrt dazu zwischen $1 / 4$ und1/3 des Einsatzes Unterzüıdkohle. Da aber gute Schwarzkoblen nngefiihr $1 / 3$ ihrer Heizkraft durch die entweichenden flüchtigen Brennstoffe verlieren, so wird auf die vorgeschlagene W'eise, durch deren Benutzung zum Rösten, das Cnterzündbrennmaterial wieder erspart. Uebrigens können auch ״um Unterzünden schlecbtere Steinkohlen ouler Braunkohlen, anch Torf gebrancht werden.

4) Ob man das Gemenge aus gleichen Theilen Erzen and Steinkohlenklein noch in Meilcrn, nach der zu St. Eticnne grebräuchlichen Weise (s. Erdmann's Journ. f. t. u. ük. Chem. B. I. S. 209) verkohlen liönne, lasse ich dahin gestellt sein.

Ceberhaupt bleibt es nun rationellen Hüttenleuten überlassen, diesen Gegenstand weiter $/ u$ verfolgen, und dabei immer zu berücksichtigen, wie viel sie in den gesinterten Massen Kohlenstoff anf die Gicht geben, um hernach zu bestimmen, wie viel noch \%. B. bei Ausübung der Methoden a und b Koblen oder Coaks den gesinterten Massen zuzugeben sind. Dass man bei jeler dieser Methoden der Sinterung auch desoxydirend auf die Metalloxydate der zu sinteruden Erze und Hüttenproducte einwirkt, und sie dadurch, einen Theil der Reductionskohle in Schaclitoren ersparend, gut vorbereitet, ist einleuchtend.

II.

Chemische Lntersuchung eines Minerulucassers von derInsel Elba, unternommen durch

W. A. L A M P A I U S, nebst einigen geognoslischen Bemerkungen über diese Insel, von

A. KRA $\mathrm{N}$ Tz.

Die Veranlassung zu der nachfolgenilen cbemischen Prófung dieses Wassers gab mir Herr August Krantza aus 